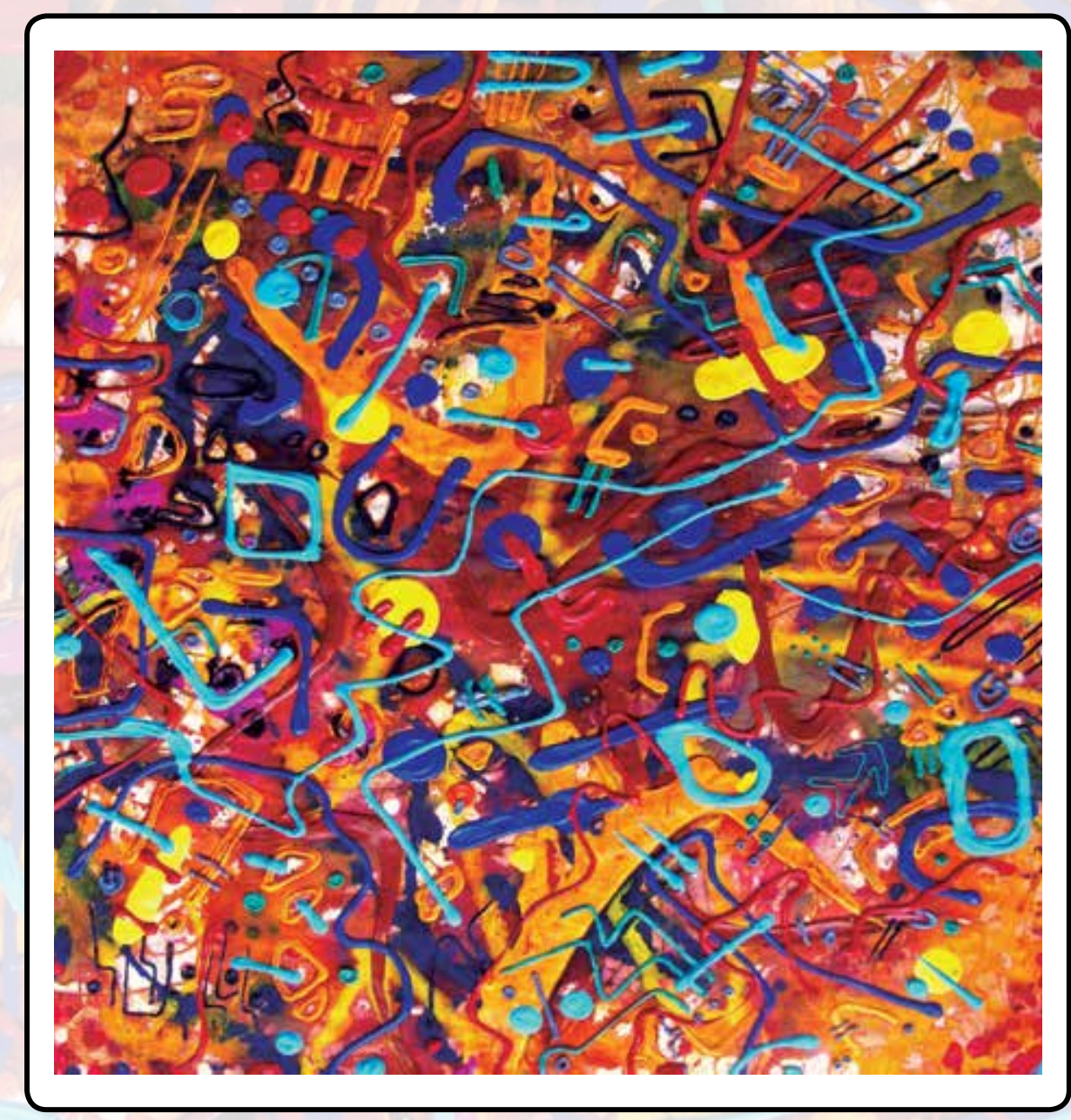

Camilo Umaña V. Sin título Acrílico sobre Lienzo 90 x80 



\title{
VARIABLES SOCIODEMOGRÁFICAS ASOCIADAS CON LA VALORACIÓN DE LAS INDICACIONES GEOGRÁFICAS COMO HERRAMIENTA DE DIFERENCIACIÓN DE LA PRODUCCIÓN AGROALIMENTARIA ${ }^{1}$
}

\author{
SOCIODEMOGRAPHICS VARIABLES ASSOCIATED WITH APPRAISAL \\ OF GEOGRAPHICAL INDICATIONS AS AGROFOOD PRODUCTS \\ DIFERENTIATION TOOLS
}

Leidy K. Garcia-Díaz ${ }^{2}$ y Wilmer S. Sepúlveda ${ }^{3}$

\begin{abstract}
Resumen
Durante los últimos años, las indicaciones geográficas han tenido un fuerte dinamismo a nivel mundial, especialmente en la zona mediterránea de Europa. Las indicaciones geográficas traen beneficios tanto a productores como a consumidores. El presente trabajo se centra en explorar los efectos diferenciadores asociados con el uso de las indicaciones geográficas en los productos agroalimentarios y se identifican variables sociodemográficas que pueden condicionar su compra. La información empleada en el estudio, proviene de la aplicación de 800 encuestas a consumidores residentes en las dos grandes ciudades del Nororiente de Colombia. En el estudio se utilizaron análisis estadísticos bivariantes. Los resultados sugieren que variables como el género, el nivel de estudios y la edad, pueden ser condicionantes de la compra de alimentos ligados a un origen geográfico.
\end{abstract}

\section{Palabras clave}

Etiqueta de calidad, indicaciones geográficas, marcas de calidad

\section{Clasificación JEL: Q13}

\begin{abstract}
During past years, geographical indications have had a strong dynamism worldwide especially in the Mediterranean zone of Europe. Geographical indications provide benefits to both, producers and consumers. This paper focuses on explore the differentiating effects associated with the use of geographical indications in agro-food products and identify the socio-demographic variables that may influence in the purchase of geographical indications products. The information used in this study comes from 800 surveys of consumers residing in the two biggest northeastern cities of Colombia. The study used bivariate statistical analysis. The results suggest that variables such as gender, education level and age, could be determinants of food purchases linked to geographical origin.
\end{abstract}

\section{Keywords}

quality label, geographical indications, quality brands

1 Artículo que presenta los resultados preliminares del proyecto "Análisis de la Designación de Origen como Herramienta de Diferenciación de la Cebolla Larga Producida en el Páramo de Berlín (Santander)”, que se ejecuta con recursos de Colciencias "Programa Jóvenes Investigadores" y la Universidad Santo Tomás, Bucaramanga (Colombia).

2 Joven Investigadora. Grupo de Investigación USTAGRI, Facultad de Administración de Empresas Agropecuarias, Universidad Santo Tomás, Bucaramanga (Colombia). Correo electrónico: ladyskatys@ hotmail.com

$3 \mathrm{Ph}$. D. en Ciencias Agrarias y del Medio Ambiente, Grupo de Investigación USTAGRI, Facultad de Administración de Empresas Agropecuarias, Universidad Santo Tomás, Bucaramanga (Colombia). 


\section{INTRODUCCIÓN}

Las indicaciones geográficas durante los últimos años han tenido un gran dinamismo a nivel mundial (Becker, 2009). En el acuerdo de la Organización Mundial del Comercio (OMC), en la ronda de Doha, sobre los aspectos de los derechos de propiedad intelectual relacionados con el comercio (ADPIC) se establece que

"las indicaciones geográficas son topónimos (...) que se utilizan para identificar productos que proceden de determinados lugares y tienen determinadas características (por ejemplo, Champagne o Roquefort"), y que "la calidad, reputación u otras características de un producto pueden determinarse en función del lugar de donde proceden".

Es importante resaltar que, por la extensión territorial derivada del número de países miembros de la OMC, el ADPIC es el acuerdo multilateral de mayor alcance en materia de indicaciones geográficas, por lo que se convierte en un referente mundial (Quintero, 2004).

Diversos gobiernos han impulsado paulatinamente la creación de diferentes mecanismos asociados con las indicaciones geográficas (marcas o etiquetas de calidad), dentro de los que sobresalen las denominaciones de origen, las indicaciones geográficas protegidas y país de origen (Becker, 2009). Las denominaciones de origen son un tipo especial de indicaciones geográficas, que se aplican a los productos cuya calidad es derivada exclusivamente del medio geográfico en el cual se producen (Organización Mundial de Propiedad Intelectual-OMPI), e. g., vino de la Rioja. Por su parte, las indicaciones geográficas protegidas, son menos explícitas que las denominaciones de origen y su calidad depende, en gran parte, del medio geográfico donde se producen (Reglamento CE No. 510/2006.), e. g., el Cordero Manchego. En relación al país de origen, esta herramienta se emplea para resaltar la calidad de un producto asociado con un territorio nacional, e.g., Café de Colombia. Debido a que la expresión "indicaciones geográficas" es de amplio espectro, en este documento se empleará de manera indistinta para designar a las denominaciones de origen, las indicaciones geográficas protegidas y el etiquetado de país de origen.

Según Arana (2005, p. 190) "Para la OMPI, las denominaciones de origen ofrecen algunas ventajas económicas, como diferenciar el producto en el mercado, facilitar la penetración del mismo en nuevos mercados y otorgarle valor agregado". Por lo cual la estrategia de diferenciación, se constituye en una herramienta útil para el posicionamiento de la producción de un país o región en los complejos mercados de la actualidad ya que, como afirma la autora precitada, condensan un conjunto de valores comerciales, de calidad y tradiciones culturales.

Diversos estudios constatan que los consumidores están dispuestos a preferir y, en ocasiones, a pagar un mayor precio por productos con determinadas características o que provienen de regiones específicas. Sin embargo, no es suficiente con la asignación de este tipo de etiquetas para conseguir la diferenciación efectiva y el 
impulso al consumo de estos productos. Se hace plenamente necesario que exista un marco institucional que respalde las medidas y que incentive la implementación de estándares de calidad, a la vez que promuevan y promocionen el consumo de los productos etiquetados (Mutter, 2006).

En Europa, líder en indicaciones geográficas que -destacando especialmente a los países del Mediterráneo- este tipo de actuaciones de diferenciación resaltan un origen geográfico, datan de hace mucho tiempo (Becker, 2009). En Francia, Italia, Portugal, España y Grecia, para el año 2010 existían registradas 904 indicaciones geográficas, distribuidas en denominaciones de origen e indicaciones geográficas protegidas (Becker, 2009). Asimismo, los países que conforman la Unión Europea parecen ser los más avanzados en el tema de la reglamentación de las indicaciones geográficas.

En Latinoamérica ya se empiezan a notar los primeros avances en el tema. Por ejemplo, la Comunidad Andina de Naciones (CAN) estableció por medio de la Decisión 486 del 2000 el Régimen Común sobre Propiedad Industrial y dedica un capítulo a las indicaciones geográficas. Por su parte, en Colombia los intentos son todavía muy tímidos. Sin embargo, existe el Decreto de la Presidencia de la República número 2591 de 2000, el Decreto de la Presidencia de la República 3081 de 2005 y la Resolución 33190 de la Superintendencia de Industria y Comercio de Octubre de 2007, por los cuales se reglamenta parcialmente la Decisión 486 de la CAN, estableciendo la autorización de uso, inscripciones y trámites vinculados a las denominaciones de origen.

Las indicaciones geográficas han mostrado ser una herramienta efectiva de diferenciación de la producción agroalimentaria. Estas pueden ser vistas como una respuesta a las preocupaciones de los consumidores por la demanda de productos "auténticos", pero más aún, son consideradas como herramientas para favorecer los intereses de los pequeños productores particulares y promover el desarrollo en áreas rurales más desfavorecidas (Dimara, Petrou \& Skuras, 2004; Sepúlveda, Maza, Pardos, Fantova \& Mantecón, 2010b). Este último aspecto cobra especial importancia en la agricultura Andina, donde se concentra un alto porcentaje de pequeños agricultores que habitan las áreas rurales. Tal como plantea Pomareda (2006 p. 2), la producción agroalimentaria con identidad cultural de territorio, que incluye los diversos tipos de designaciones de origen geográfico, se han reconocido como herramientas que pueden generar más ingresos para los productores rurales. Estas consideraciones permiten destacar la importancia de investigaciones que apunten a evaluar las posibilidades de utilización de estos métodos de diferenciación de la producción agroalimentaria en nuestro país.

Con relación a los consumidores, dentro de los atributos de calidad de confianza, el origen es uno de los aspectos más demandados por ellos (Bernués, Olaizola \& Corcoran, 2003; Sepúlveda, Maza \& Mantecón, 2008). Desde la perspectiva de la economía de la información, los atributos de calidad de confianza son aquellos que 
no pueden ser conocidos ni antes ni después de la compra, o pueden serlo a un costo muy elevado (Sepúlveda et al., 2008). En este caso, el empleo de una marca (marca de calidad) asociada a una indicación geográfica es de gran importancia para señalar el origen de los productos y resaltar la calidad ante los consumidores, ya que permite transformar atributos de calidad de experiencia en atributos de calidad de búsqueda, es decir, aquellos que pueden ser evaluados antes de la compra (Busch, Thiagarajan, Hatanaka, Bain, Flores \& Frahm, 2005; Sepúlveda et al., 2008).

Estas marcas de calidad, pueden representar algún valor para los consumidores puesto que ellos las pueden percibir como una señal particular de especificaciones del producto y de su nivel de calidad (Verbeke \& Roosen, 2009). Existen diversos estudios, donde se ha podido corroborar que el lugar de origen tiene gran importancia para los consumidores y además influye positivamente en el proceso de elección del consumidor (Bello \& Calvo, 1998; Bernués, et al. 2003; Sánchez, Sanjuán \& Akl, 2001). Algunas variables sociodemográficas como la edad, nivel educativo y el nivel de renta, han sido identificadas como potencialmente asociadas con la compra de productos diferenciados ligados con un origen geográfico y con etiquetas de calidad (Angulo, Gil \& Tamburo, 2005; Sánchez, Beriain \& Carr, 2012; Sepúlveda et al., 2008; Sepúlveda, Maza \& Mantecón, 2010a; Wachenheim, Alonso \& Dumler, 2000). La mayoría de estos estudios se concentran en Europa, aunque se han reportado algunos trabajos recientes en Latinoamérica (Villalobos, Padilla, Ponce \& Rojas, 2010; Schnettler, Ruiz \& Sepúlveda, 2007; Schnettler, Zavala \& Pihan, 2009; Schnettler, Cid, Miranda, Sepúlveda \& Denegri, 2010a; Schnettler et al., 2010b; Padilla, Villalobos, Spiller \& Henry, 2007), principalmente en Chile. En Colombia, estudios que analicen las indicaciones geográficas desde el punto de vista del consumidor y las variables personales ligadas con la compra de productos diferenciados ligados con el origen son limitados.

En este contexto, el presente trabajo se centra en analizar desde el punto de vista de los consumidores, aspectos asociados con el efecto diferenciador que pueden suponer las denominaciones de origen en los productos agroalimentarios en el Nororiente de Colombia, que identificando las variables sociodemográficas y que pueden condicionar su compra en esta parte del país.

\section{METODOLOGÍA}

La información contenida en el presente estudio, proviene de la realización de 800 entrevistas personales basadas en encuestas (cara a cara), llevadas a cabo en las ciudades de Bucaramanga y Cúcuta, los dos centros urbanos con mayor población ubicados en el Nororiente de Colombia. Desarrollar el estudio en esta zona del país es especialmente relevante ya que, productos de dicha región como la piña de Lebrija, el bocadillo veleño, hormigas culonas y las cebollas ocañeras, han sido identificados como potenciales candidatos sobre los cuales se puede tramitar una denominación 
de origen (Departamento Nacional de Planeación - DNP, 2006). El muestreo de tipo aleatorio simple llevado a cabo en ambas ciudades para la recolección de la información, fue realizado en el segundo semestre del año 2012. En cada ciudad, asignando a p y q valores intermedios y para un intervalo de confianza en los resultados del $95.5 \%(Z=2)$, el máximo error que se pudo cometer fue del $5 \%$. La descripción de la muestra se presenta en la Tabla 1.

La muestra estuvo conformada en un mayor porcentaje por mujeres con un $59.6 \%$ mientras que los hombres representaron el $40.4 \%$ de las personas encuestadas (ver Tabla 1). Con relación a la edad de los encuestados, las personas con edades iguales o menores a 20 años representaron el $15.1 \%$, con edades entre 21 y 30 años correspondieron con el $30.5 \%$, entre 31 y 45 años tuvieron una participación del $26.9 \%$, en el rango de edad de 46 a 60 años representaron el $20.6 \%$ y el rango de mayores de 60 años, se correspondió con un $6.9 \%$ del tamaño de la muestra.

Con relación al nivel de formación académica, el 19.0\% de los encuestados poseía estudios básicos / primarios, el $32.7 \%$ bachillerato, el $25.2 \%$ estudios técnicos o tecnológicos y el $23.1 \%$ estudios superiores. Por otro lado, y con relación al nivel de ingresos mensuales recibidos en los hogares a los que pertenecían los encuestados, el 36.3\% manifestó que poseía ingresos mensuales en sus hogares inferiores a $\$ 1.000 .000$, el $21.0 \%$ entre $\$ 1.000 .000$ y $\$ 1.500 .000$, el $18.9 \%$ entre $\$ 1.500 .000$ y $\$ 2.500 .000$ y el $12.5 \%$ poseía ingresos superiores a $\$ 2.500 .000$; el $11.3 \%$ restante manifestó no saber o no responder a esta pregunta.

\begin{tabular}{|l|l|}
\hline \multicolumn{2}{|c|}{ Tabla 1. Características sociodemográficas de la muestra } \\
\hline Variables Sociodemográficas & \multicolumn{1}{c|}{ Porcentaje $\%$} \\
\hline Hénero de la persona encuestada & \\
\hline Mujer & $40.4 \%$ \\
\hline & $59.6 \%$ \\
\hline Rango de Edad & \\
\hline Igual o menor a 20 años & \\
\hline Entre 21 y 30 años & $15.1 \%$ \\
\hline Entre 31 y 45 años & $30.5 \%$ \\
\hline Entre 46 y 60 años & $26.9 \%$ \\
\hline Más de 60 años & $20.6 \%$ \\
\hline & $6.9 \%$ \\
\hline
\end{tabular}




\begin{tabular}{|l|c|}
\hline Nivel de Estudios & \\
\hline Básico - Primaria & $19.0 \%$ \\
\hline Bachillerato & $32.7 \%$ \\
\hline Técnico - Tecnológico & $25.2 \%$ \\
\hline Superior (Universitario) & $23.1 \%$ \\
\hline
\end{tabular}

\begin{tabular}{|l|c|}
\hline Rango de Ingresos (mes) & \\
\hline Menos de $\$ 1.000 .000 /$ mes & $36.3 \%$ \\
\hline Entre $\$ 1.000 .000$ y $\$ 1.500 .000 /$ mes & $21.0 \%$ \\
\hline Entre $\$ 1.500 .000$ y $\$ 2.500 .000 /$ mes & $18.9 \%$ \\
\hline Más de $\$ 2.500 .000 /$ mes & $12.5 \%$ \\
\hline No sabe / No contesta & $11.3 \%$ \\
\hline
\end{tabular}

Elaboración de los autores

En la encuesta ómnibus de tipo cerrada, se incluyeron varios tipos de preguntas. Un bloque de preguntas incluyó variables sociodemográficas como el género, el nivel de estudios alcanzado, el rango de edad y el rango de ingresos mensuales. Con el fin de identificar el efecto diferenciador que puede suponer ante los consumidores el origen geográfico de los productos agroalimentarios, se empleó una batería de preguntas compuesta por cuatro afirmaciones, medidas sus respuestas a través de una escala Likert, donde el número 1 indicaba estar totalmente en desacuerdo, el número 2 estar en desacuerdo, el número 3 estar ni de acuerdo ni desacuerdo, el número 4 estar de acuerdo y el número 5 estar totalmente de acuerdo frente a cada afirmación planteada.

Las cuatro afirmaciones planteadas en la pregunta referente a la valoración del lugar de origen de los productos agroalimentarios, fueron establecidas de acuerdo a la literatura reportada. La afirmación "el lugar de origen me indica el nivel de calidad de un producto" fue incluida debido a que diversos autores (Schnettler et al., 2007; Sepúlveda et al., 2008), han planteado que el lugar de origen de los productos tiene un efecto significativo en la evaluación de calidad de los productos durante el proceso de decisión de compra. Por su parte, la afirmación "el lugar de origen siempre debe estar visible en la etiqueta", fue incluida en la batería de preguntas, debido a que una forma clara de hacer tangible el suministro de la información sobre el lugar de origen hacia los consumidores, es a través de una marca de calidad incluida en la etiqueta de los productos, casi siempre efectuada por una certificación de tercera parte (Busch 
et al., 2005; Caswell, 1998). El empleo de una marca o sello de calidad, permite transformar un atributo de calidad de creencia como es el origen, en atributos de calidad de búsqueda (Caswell, 1998).

La inclusión de la afirmación "el lugar de origen me indica que el producto posee características que lo hacen diferente a los demás", se debe a que la percepción de la zona de origen de un alimento por parte de los consumidores, se ha usado activamente para promocionar las características diferenciadoras, condiciones naturales y tradiciones artesanales asociadas con un producto, cuya calidad superior está fuertemente asociada con el medio geográfico donde tienen lugar (Schnettler et al., 2009; Pérez \& Bustamante, 1999). Asimismo, se ha podido determinar que la imagen de la zona de origen de un producto, le confiere una identidad única y genera valor agregado (Schnettler et al., 2009). Por último, la afirmación "me genera confianza en el momento de la compra", fue incluida debido a que el origen geográfico actúa como indicativo de confianza, sobretodo donde los estándares asociados con los sistemas de producción de las denominaciones de origen legalmente reconocidas, son generadores de seguridad hacia los consumidores (Sepúlveda et al, 2008).

Para el análisis estadístico de los datos se empleó el software SPSS versión 15.0. Los análisis estadísticos consistieron fundamentalmente en análisis bivariantes, esto con el fin de identificar las relaciones existentes entre las cuatro afirmaciones expuestas en el formulario y las variables sociodemográficas. Los análisis bivariantes empleados fueron el análisis de varianza (ANOVA), Test de comparación de medias (t de Student) y Tablas de contingencia con pruebas de Chi-cuadrado (Glantz, 2006).

\section{RESULTADOS Y DISCUSIÓN}

Los resultados relativos a las valoraciones de los encuestados frente a las cuatro afirmaciones, se muestran en la Figura 1. La afirmación el lugar de origen me genera confianza al momento de la compra del producto, obtuvo una valoración promedio de 4.12, ubicándose en el primer lugar. Por su parte, la afirmación el lugar de origen, me indica que ese producto posee características que lo hacen diferente a los demás, se situó en el segundo lugar, con una valoración media de 3.93. La afirmación el lugar de origen, siempre debe estar visible en la etiqueta del producto ó en el lugar de compra del producto, obtuvo una valoración media de 3.92 ocupó el tercer lugar; $\mathrm{y}$, en el cuarto lugar, con una valoración de 3.81, se ubicó la afirmación el lugar de origen me indica el nivel de calidad de un producto. Todas las afirmaciones poseen valoraciones considerables, lo que deja ver la importancia que podrían suponer las denominaciones de origen como herramienta de diferenciación de la producción. Los resultados están acordes con la literatura (Schnettler et al., 2007; Caswell, 1998; Schnettler et al., 2009; Pérez \& Bustamante, 1999). Estos resultados son relevantes, ya que si bien es cierto que este tipo de estudios son muy limitados en el territorio nacional, parece ser que la literatura sobre las denominaciones de origen tienen un 
alto grado de aplicabilidad en nuestro contexto, lo que podrían suponer una mejora en las actuaciones de las entidades públicas y privadas de cara a una mejor adopción de las mismas.

Figura 1. Efecto diferenciador de las denominaciones de origen

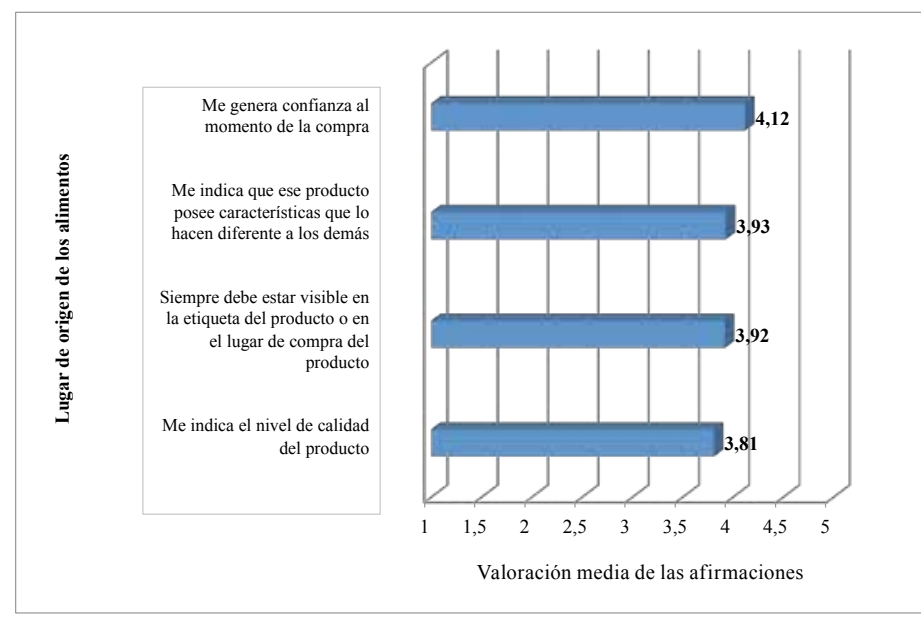

Elaboración de los autores

Tenidas en cuenta las cuatro afirmaciones, se encontró diferencia significativa en la valoración media efectuada entre hombres y mujeres (T Student, $P<0.1$ ) a la visibilidad del lugar de origen en la etiqueta de los productos. Esto sugiere, que aunque las demás afirmaciones incluidas en el bloque de preguntas (confianza al momento de la compra, características del producto que lo hacen diferente de los demás y es un indicativo del nivel de calidad del producto) son valoradas de forma similar tanto por hombres como por mujeres (ver Figura 2), las mujeres le otorgan una mayor importancia a la disponibilidad del lugar de origen geográfico plasmado en la etiqueta, lo cual sugiere que éste sea un factor que podría influenciar su proceso/ decisión de compra. Respecto al uso de las etiquetas en los alimentos, investigaciones han reportado que las mujeres respecto a los hombres le asignan mucha más importancia a esta señal de calidad extrínseca (Wandel, 1997; Verbeke \& Ward, 2006), por lo que los resultados obtenidos en esta investigación son consistentes con la literatura. Según Rimal (2005), esta mayor importancia por emplear las etiquetas por parte de las mujeres, se acentúa cuando ellas poseen mayores niveles académicos y una dieta saludable. 
Figura 2. Valoraciones del lugar de origen vs. género.

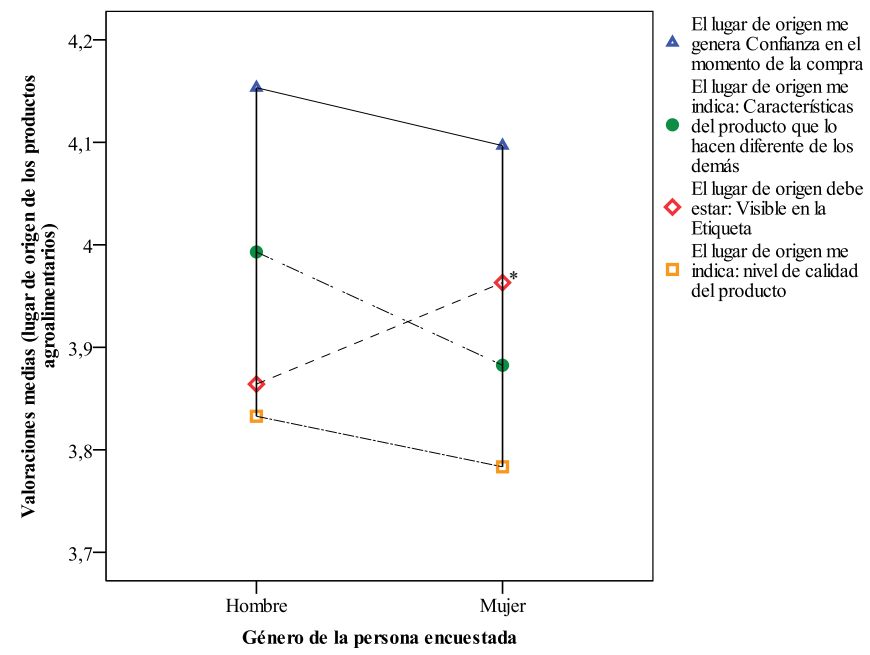

Nota: $*=P<0.1$ para el t de Student. Elaboración de los autores

Con relación al nivel de estudios alcanzado por los encuestados, se hallaron diferencias significativas (ANOVA, $P<0.05$ ) entre éste y las valoraciones medias asignadas a las cuatro afirmaciones relacionadas con el lugar de origen de los productos agroalimentarios incluidas en el cuestionario (ver Figura 2). Al entrar más en detalle, se observa que las personas con menor nivel educativo, son aquellas que le asignan una mayor valoración a todas las variables presentadas. Un aspecto para resaltar, es la baja valoración asignada por los niveles de estudios bachillerato, técnico-tecnólogo y superior hacía el lugar de origen como un indicativo de calidad del producto, mientras que las personas ubicadas en dichos niveles, asocian más el lugar de origen de los productos con la confianza que le genera al momento de la compra y con posibles características que lo hacen diferente de los demás. Al analizar estas mismas variables con los rangos de edad, se encuentra una relación significativa inversa, en el sentido que las personas con mayores rangos de edad son las que valoran en mayor medida estos cuatro aspectos, mientras que las personas ubicadas en los rangos de edad inferiores, le otorgan menos valoraciones, con diferencias significativas en tres de ellas (ANOVA, $P<0.05$ ) (ver Figura 3). Esta relación doble inversa, podría deberse a que las personas con mayores niveles de edad son las que poseen menores niveles de formación académica (Chi-cuadrado, $P=0.000)$. 
Figura 3. Valoraciones del lugar de origen vs. nivel de estudios

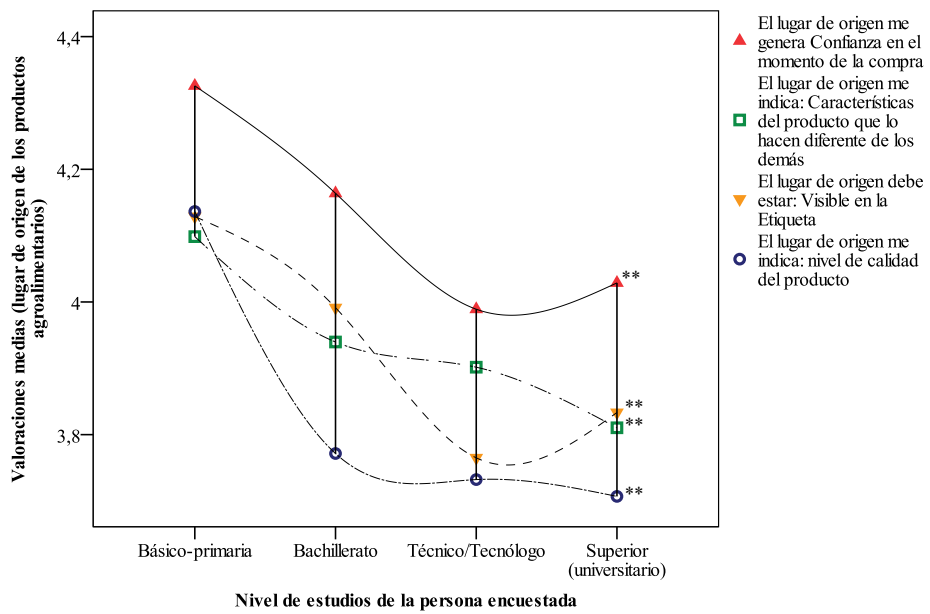

Nota: $* *=P<0.05$ para el ANOVA.

Fuente: Elaboración de los autores

Figura 4. Valoraciones del lugar de origen vs. rango de edad

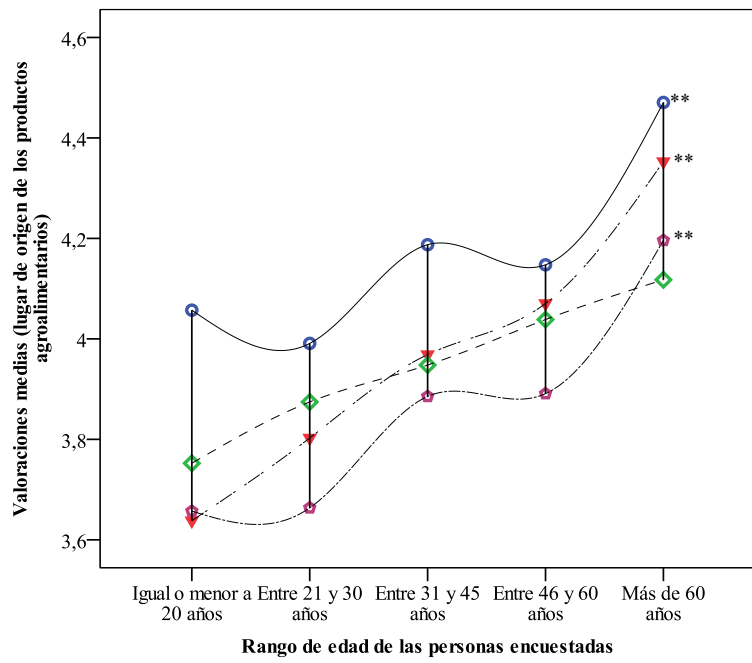

El lugar de origen me

- genera Confianza en el momento de la compra El lugar de origen debe $\checkmark$ estar: Visible en la Etiqueta El lugar de origen me indica: Características $\checkmark$ del producto que lo hacen diferente de los demás

El lugar de origen me

- indica: nivel de calidad del producto

Nota: $* *=P<0.05$ para el ANOVA.

Fuente: Elaboración de los autores 
De acuerdo con Verbeke, Demey, Bosmans y Viaene (2005), esta valoración alta por parte de las personas mayores, podría deberse a que ellas poseen mayor apego a los productos tradicionales, lo que al final se traduce en una mayor valoración del origen como indicativo de calidad superior. Asimismo Sepúlveda et al. (2010) a y Verbeke y Ward (2006), encontraron que las personas con mayores rangos de edad en contraposición con los más jóvenes, eran los más predispuestos a adquirir productos agroalimentarios con etiquetas de calidad de origen. Schnettler et al., 2007 sugieren, que las personas más jóvenes poseen menos vínculos con la tradicionalidad de alimentos diferenciados, lo que los haría menos proclives a demandar el lugar de origen en la etiqueta de los alimentos que ellos adquieren. Otro aspecto que podría ayudar a explicar esta baja valoración por parte de los jóvenes, es que las personas con menores edades tienden a ser menos etnocentristas y más cosmopolitas que las de mayor edad (Schnettler et al., 2007; Clemente, Rodríguez \& Buitrago, 2011).

Relativo al nivel de estudios, parece existir ciertas contraposiciones con los resultados obtenidos en el presente estudio, en el sentido que Sánchez et al., 2012, en un estudio llevado a cabo en España, encontraron que las personas con mayores niveles de estudios le otorgaban mayor importancia a la disponibilidad del lugar de origen en la compra de alimentos. Sin embargo, sus resultados podrían deberse a la gran presencia de personas con altos niveles académicos que caracterizó la muestra empleada.

Con relación a los niveles de ingresos mensuales, no se encontraron diferencias significativas entre esta variable y las valoraciones medias efectuadas por los encuestados a las cuatro variables objeto (ANOVA, $P<0.05$ ). Esto sugiere, que al parecer las personas, sin importar el rango de ingresos mensuales que perciben, le asignan la misma importancia a las variables relativas al lugar de origen de los alimentos, como factor que podría influenciar el proceso de compra. Relativo a esta variable, a través de diversos estudios se ha encontrado diversas contraposiciones. Por ejemplo, mientras que Angulo et al. (2005), Sánchez et al. (2012) y Sepúlveda et al. (2008) encontraron que las personas con mayores niveles de renta eran más predispuestas a adquirir productos vinculados con un origen geográfico, Martínez, Hanagriff, Lau y Harris (2007), encontraron que el nivel de renta no ejerce un efecto significativo en la adquisición de productos diferenciados.

\section{Conclusiones e implicaciones futuras}

Este trabajo llevado a cabo en el nororiente de Colombia, se centró en la exploración de los efectos diferenciadores asociados con el uso de las indicaciones geográficas en los productos agroalimentarios y se identificaron variables sociodemográficas que pueden condicionar su compra. El género, la edad y el nivel de estudios, se mostraron como variables que potencialmente pueden afectar la compra de productos diferenciados de calidad. Por tanto, se deberían tener en cuenta estos 
resultados, de cara al desarrollo de estrategias de marketing dirigidas a segmentos específicos de consumidores, para así lograr un mayor impacto comercial de los productos agroalimentarios tradicionales en los que se utilicen estas herramientas de diferenciación de la producción.

Por otra parte, es interesante resaltar, que aunque en Colombia parece existir una base legal sólida, se encuentran registradas pocas marcas de productos agroalimentarios asociadas con indicaciones geográficas (DNP, 2010). Esto hace suponer que los entes gubernamentales, poco han incentivado la adopción de indicaciones geográficas y desaprovechan el potencial que posee el país en la materia (DNP, 2010). Hay que tener presente que Colombia, recientemente ha firmado importantes Tratados de Libre Comercio. En el caso de la Unión Europea, es imprescindible poder adoptar las mejores prácticas en torno a la gestión de indicaciones geográficas, ya que son ellos quienes han demostrado mayor dinamismo y desarrollo en este campo. Sería interesante poder identificar más a profundidad, el efecto diferenciador que pueden suponer los productos agroalimentarios regionales frente a los importados, de cara a poder generar herramientas dirigidas a los pequeños productores, para que puedan, al menos en parte, blindar sus producciones y continuar ejerciendo su función dinamizadora del mundo rural.

\section{Agradecimientos}

El trabajo de investigación se desarrolló gracias a la ayuda concedida por el Departamento Nacional de Ciencia y Tecnología-COLCIENCIAS programa Jóvenes Investigadores a L.K. García-Díaz. Asimismo, los autores desean agradecer la ayuda económica suministrada por la Universidad Santo Tomás Bucaramanga, mediante el proyecto "Análisis de la Designación de Origen como Herramienta de Diferenciación de la Cebolla Larga Producida en el Páramo de Berlín (Santander)". Los autores quieren agradecer igualmente, al Centro de Productividad y Competitividad del Oriente por su apoyo en la recolección de la información.

\section{REFERENCIAS}

Angulo, A.M., Gil, J.M., \& Tamburo, L. (2005). Food safety and consumers willingness to pay for labelled beef in Spain. Journal of Food Products Marketing, 11(3), 89-105.

Arana, M.C. (2005). Las Denominaciones de Origen: una metodología para su reconocimiento. Revista de la Competencia y la Propiedad Intelectual 1(1), 189212.

Becker, T. (2009). European food quality policy: the importance of geographical indications, organic certification and food quality assurance schemes in European 
countries. The Estey Centre Journal of International Law and Trade Policy, 10(1), 111-130.

Bello, L., \& Calvo, D. (1998). Propuesta de un modelo positivo del proceso de compra de carne de ternera y evaluación de las preferencias de los consumidores. Revista Española de Economía Agraria, 183, 201-220.

Bernués, A., A. Olaizola, \& K. Corcoran. (2003). Extrinsic attributes of red meat as indicators of quality in Europe: an application for market segmentation. Food Quality and Preference, 14, 265-276.

Busch, L., Thiagarajan, D., Hatanaka, M., Bain, C., Flores, L.G., \& Frahm, M. (2005). The relationship of third-party certification (TPC) to sanitary/phytosanitary (SPS) measures and the international agri-food trade. United States: United States Agency for International Development, Raises SPS Global Analytical Report 9.

Caswell, J. (1998). How labeling of safety and process attributes affects markets for food. Agricultural and Resource Economics Review, 27(2), 151-158.

Clemente, R., Rodríguez, B. \& Buitrago, V. (2011). Importancia del origen geográfico en el consumo de productos agroalimentarios. Scripta Nova, 15(369).

Decisión 486 de 2000 sobre el régimen común de Propiedad Industrial de la Comunidad Andina de Naciones. Disponible en: http://www. comunidadandina. org/normativa/ dec/ D486.htm. Recuperado en junio de 2012.

Decreto número 2591/ 2000. República de Colombia. Por el cual se reglamenta parcialmente la Decisión 486 de la Comisión de la Comunidad Andina.

Decreto número 3081/2005. República de Colombia. Por el cual se reglamenta parcialmente la Decisión 486 de la Comunidad Andina.

Departamento Nacional de Planeación (DNP) (2010). Plan nacional de desarrollo 2010-2014.

Departamento Nacional de Planeación (DNP). (2006). Estudio para la identificación de los productos potenciales en los sectores agropecuarios, agroindustrial y artesanal, que podrían ser protegidos a través de denominaciones de origen, marcas colectivas o certificación. Disponible en: http://www.dnp.gov.co/ LinkClick.aspx?fileticket=IT4oo7MxS6g\%3D\& tabid=89

Dimara, E., Petrou, A., \& Skuras, D. (2004). Agricultural policy for quality and producers' evaluations of quality marketing indicators: a Greek case study. Food Policy, 29, 485-506.

Glantz, S.A. (2006). Bioestadística (6ª ed.), México: McGraw-Hill Interamericana. 
Martínez, S., Hanagriff, R., Lau, M., \& Harris, M. (2007). Factors affecting demand for branded beef. In Paper presented at the 39th annual meetings program southern agricultural economics association, February 4-6 2007, Mobile, United States.

Mutter, K. (2006). Propiedad intelectual y desarrollo en Colombia. Revista Estudios Socio-Jurídicos, 8(2), 85-101.

Organización Mundial del Comercio (OMC). (2012). Disponible en: http://www. wto.org/spanish/tratop_s/trips_s/gi_background_s.htm, leído en julio de 2012.

Organización Mundial de la Propiedad Intelectual (OMPI). (2012). Disponible en: http://www.wipo.int/about-ip/es/about_geographical_ind.html\#P20_1291, leído en julio de 2012.

Padilla, C., Villalobos, P., Spiller, A. \& Henry, G. (2007). Preferencia y disposición del consumidor a pagar por un sello de calidad certificada: Implicancias para productores de alimentos de origen campesino. Agricultura Técnica, 67(3), 300308.

Pérez, G. \& Bustamante, I. (1999). Las denominaciones de origen e indicaciones geográficas en la Unión Europea: su libre circulación y consideración de signos distintivos de calidad. Economía Aragonesa, 104-126.

Pomareda, C. (2006). Factores estructurales y políticas que limitan o favorecen la producción agroalimentaria con identidad territorial. In Ponencia presentada en el III Congreso Internacional de la Red SIAL "Sistemas Agroalimentarios Locales" Alimentación y Territorios "ALTER.

Quintero, A. (2004). Visión de las denominaciones de origen en Colombia. REVIST@e-Mercatoria,3(1),1-14.

Reglamento (CE) No. 510/2006 sobre la protección de las indicaciones geográficas y las denominaciones de origen de los productos agrícolas y alimenticios.

Resolución 33190 de Octubre de 2007. República de Colombia. Ministerio de Comercio, Industria y Turismo Superintendencia de Industria y Comercio.

Rimal, A. (2005): Meat labels: consumer attitude and meat consumption pattern. International Journal of Consumer Studies, 29(1), 47-54.

Sánchez, M., Beriain, M.J., \& Carr, T.R. (2012). Socio-economic factors affecting consumer behaviour for United States and Spanish beef under different information scenarios. Food Quality and Preference, 24, 30-39

Sánchez, M., Sanjuán, A. I., \& Akl, G. (2001). El distintivo de calidad como indicador de seguridad alimenticia en carne de vacuno y cordero. Economía Agraria y Recursos Naturales, 1(1), 77-94.

Schnettler, B., Ruiz, D., \& Sepúlveda O. (2007). Importancia del origen en el consumo de alimentos en la IX Región de Chile. Idesia, 25(3), 19-29. 
Schnettler, B., Zavala, C. \& Pihan, R. (2009). Influencia de la zona de origen en la compra de hortalizas en la IX Región de Chile. Idesia, 27(1), 13-22.

Schnettler, B., Cid, E., Miranda, H., Sepúlveda, J. \& Denegri, M. (2010)a. Etnocentrismo y Efecto País de Origen en la Compra de Arroz en Supermercados de las Ciudades de Los Ángeles y Temuco, Chile. Panorama Socioeconómico, 40, 18-33.

Schnettler, B., Obreque, B., Cid, E., Mora, M., Miranda, H., Sepúlveda, J. \& Denegri, M. (2010)b. Influencia del país de origen en la toma de decisión de compra de alimentos: un estudio en consumidores de distinta etnia en Chile. Revista Latinoamericana de Psicología, 42(1), 119-130.

Sepúlveda, W.S., Maza, M.T., \& Mantecón, A.R. (2008). Factors that affect and motivate the purchase of quality-labelled beef in Spain. Meat Science, 80, 12821289.

Sepúlveda, W.S., Maza, M.T., \& Mantecón, A.R. (2010)a. Factors associated with the purchase of designation of origin lamb meat. Meat Science, 85, 167-173.

Sepúlveda, W.S., Maza, M.T., Pardos, L., Fantova, E., \& Mantecón, A.R. (2010)b. Farmers' attitudes towards lamb meat production under a protected geographical indication. Small Ruminant Research, 94, 90-97.

Verbeke, W., Demey, V., Bosmans, W., \& Viaene, J. (2005). Consumer versus producer expectations and motivations related to "superior" quality meat: qualitative research findings. Journal of Food Products Marketing, 11(3), 27-41.

Verbeke, W. \& Roosen, J. (2009). Market differentiation potential of country-oforigin, quality and traceabilitylLabeling. Estey Centre Journal of International Law and Trade Policy, 10(1), 20-35.

Verbeke, W., \& Ward, R. (2006). Consumer interest in information cues denoting quality, traceability and origin: An application of ordered probit models to beef labels. Food Quality and Preference, 17, 453-467

Villalobos, P., Padilla, C., Ponce, C. \& Rojas, A. (2010). Beef consumer preferences in Chile: Importance of quality attribute differentiators on the purchase decision. Chilean Journal Of Agricultural Research, 70(1), 85-94.

Wachenheim, C., Alonso, C., \& Dumler, M. (2000). Marketing a branded fresh beef product. Journal of Food Products Marketing, 6 (1), 53-79.

Wandel, M. (1997): Food labelling from a consumer perspective. British Food Journal, 9(6), 212. 